UA/NPPS-7-02

\title{
BOOTSTRAPING THE QCD CRITICAL POINT
}

\author{
N. G. Antoniou and A. S. Kapoyannis \\ Department of Physics, University of Athens, 15771 Athens, Greece
}

\begin{abstract}
It is shown that hadronic matter formed at high temperatures, according to the prescription of the statistical bootstrap principle, develops a critical point at nonzero baryon chemical potential. The location of the critical point in the phase diagram, however, depends on the detailed knowledge of the partition function of the deconfined phase, near the critical line. In a simplified version of the quark-gluon partition function, the resulting location of the critical point is compared with the solutions of other approaches and in particular with the results of lattice QCD. The proximity of our solution to the freeze-out area in heavy-ion experiments is also discussed.
\end{abstract}

PACS numbers: 12.40.Ee

Keywords: Statistical Bootstrap, QCD Critical Point, QCD phase diagram 


\section{Introduction}

Critical QCD at nonzero baryon density and high temperature is a theory of fundamental significance associated with the properties of primordial matter (quark matter) both in the early universe and in the multiparticle environment, created in heavy-ion collisions. Theoretical arguments, based on the universal features of chiral phase transition, suggest with confidence the existence of a critical point in QCD which is the end point of a quark-hadron critical line of first order and belongs to the Ising universality class [1]. The location of the critical point in the phase diagram needs a more detailed treatment, based on first principles and it is only recently that a first attempt to locate the critical point, within the framework of lattice QCD, has appeared [2]. It is of interest to note that the knowledge of the location of the critical point in the phase diagram is of primary importance for heavy-ion physics since one expects to observe spectacular critical fluctuations only in those collisions of nuclei which freeze-out close to the critical point [3].

In this Letter we present a different, complementary approach in the search for the existence and location of the critical point, based on the statistical bootstrap principle [4-6]. We notice that in the vicinity of the QCD critical point (in the crossover regime) the hadronic degrees of freedom are thermodynamically indistinguishable from the quark matter degrees of freedom, suggesting that the QCD critical point must also be present in the solution associated with the statistical bootstrap equations. These equations describe the dynamics of interacting hadronic matter at high temperatures, incorporating in the partition function the observed hadronic spectrum [4-6].

In the context of the Statistical Bootstrap the interaction among hadrons is simulated by the presence of clusters. The bootstrap hypothesis adopts that every cluster is composed by a number of other clusters described by the same mass spectrum $\tilde{\tau}\left(m^{2}, b, s, \ldots\right)$, where $b$ stands for baryon number, $s$ for strangeness and the dots for any additional quantum number that can characterise a cluster, like charge $q$, absolute strangeness (number of strange plus antistrange quarks) $|s|$, etc. Then $\tilde{\tau} d m^{2}$ equals to the number of clusters with mass $m$ and quantum numbers equal to the set $b, s, \ldots$ Every cluster is also characterised by a kinematical term $\tilde{B}\left(m^{2}\right)$, apart from its mass spectrum which is of dynamical origin. The conservation of four-momentum, as well as, conservation of quantum numbers can be 
accommodated by a suitable bootstrap equation [7]

$$
\begin{gathered}
\tilde{B}\left(p^{2}\right) \tilde{\tau}\left(p^{2}, b, s, \ldots\right)=\underbrace{g_{b, s, \ldots} \tilde{B}\left(p^{2}\right) \delta_{0}\left(p^{2}-m_{b, s, \ldots}^{2}\right)}_{\text {input term }}+\sum_{n=2}^{\infty} \frac{1}{n !} \int \delta^{4}\left(p-\sum_{i=1}^{n} p_{i}\right) . \\
\cdot \sum_{\left\{b_{i}\right\}} \delta_{K}\left(b-\sum_{i=1}^{n} b_{i}\right) \sum_{\left\{s_{i}\right\}} \delta_{K}\left(s-\sum_{i=1}^{n} s_{i}\right) \ldots \prod_{i=1}^{n} \tilde{B}\left(p_{i}^{2}\right) \tilde{\tau}\left(p_{i}^{2}, b_{i}, s_{i}, \ldots\right) d^{4} p_{i} .
\end{gathered}
$$

The pattern of clusters composed of clusters continues until the known hadrons, considered as the "input" particles, are reached. The bootstrap equation after performing a series of Laplace transformations acquires the form ${ }^{1}$.

$$
\varphi(\beta,\{\lambda\})=2 G(\beta,\{\lambda\})-\exp [G(\beta,\{\lambda\})]+1
$$

In the last equation $G(\beta,\{\lambda\})$ is the Laplace transform of the mass spectrum with the accompanying kinematical term

$$
\begin{gathered}
G(\beta,\{\lambda\})=\sum_{b^{\prime}=-\infty}^{\infty} \lambda_{b}^{b^{\prime}} \sum_{q^{\prime}=-\infty}^{\infty} \lambda_{q}^{q^{\prime}} \sum_{s^{\prime}=-\infty}^{\infty} \lambda_{S}^{s^{\prime}} \sum_{\left|s^{\prime}\right|=0}^{\infty} \gamma_{s}^{\left|s^{\prime}\right|} \int e^{-\beta^{\mu} p_{\mu}} \tilde{B}\left(p^{2}\right) \tilde{\tau}\left(p^{2}, b^{\prime}, q^{\prime}, s^{\prime},\left|s^{\prime}\right|\right) d p^{4}, \\
=\frac{2 \pi}{\beta} \int_{0}^{\infty} m \tilde{B}\left(m^{2}\right) \tilde{\tau}\left(m^{2},\{\lambda\}\right) K_{1}(\beta m) d m^{2}
\end{gathered}
$$

and $\varphi(\beta,\{\lambda\})$ the Laplace transform of the input term ${ }^{2}$

$$
\varphi(\beta,\{\lambda\})=\sum_{b^{\prime}=-\infty}^{\infty} \lambda_{b}^{b^{\prime}} \sum_{q^{\prime}=-\infty}^{\infty} \lambda_{q}^{q^{\prime}} \sum_{s^{\prime}=-\infty}^{\infty} \lambda_{S}^{s^{\prime}} \sum_{\left|s^{\prime}\right|=0}^{\infty} \gamma_{s}^{\left|s^{\prime}\right|} \int e^{-\beta^{\mu} p_{\mu}} g_{b^{\prime} q^{\prime} s^{\prime}\left|s^{\prime}\right|} \tilde{B}\left(p^{2}\right) \delta_{0}\left(p^{2}-m_{b^{\prime} q^{\prime} s^{\prime}\left|s^{\prime}\right|}^{2}\right) d p^{4} .
$$

The masses $m_{b q s|s|}$ correspond to the masses of the input particles, which in this paper will be all the known hadrons with masses up to $2400 \mathrm{MeVs}$, the $g_{b q s|s|}$ are degeneracy factors due to spin and the $\lambda$ 's are the fugacities of the input particles.

The bootstrap equation [7] defines the boundaries of the hadronic phase through the relation

$$
\varphi(\beta,\{\lambda\}) \leq \ln 4-1
$$

\footnotetext{
${ }^{1}$ We shall use the abbreviation $\{\lambda\}$ for the set of fugacities corresponding to the quantum numbers $(b, q, s,|s|)$, i.e. $\left(\lambda_{b}, \lambda_{q}, \lambda_{S}, \gamma_{s}\right)$.

${ }^{2}$ The Statistical Bootstrap Model is formulated in the Boltzmann approximation which is acceptable throughout the phase diagram except for low temperatures and high baryon densities, where the effect of the Bose-Einstein and Fermi-Dirac statistics becomes important [5]. For example for the temperature and baryon density which correspond to SPS the error due to the neglection of the correct statistics does not exceeds $3 \%[7]$.
} 
The same equation leads to the asymptotic mass spectrum of interacting hadrons

$$
\tilde{\tau}\left(m^{2},\{\lambda\}\right) \stackrel{m \rightarrow \infty}{\longrightarrow} 2 C(\{\lambda\}) m^{-\alpha-1} \exp \left[m \beta^{*}(\{\lambda\})\right]
$$

where $\beta=T^{-1}$ and $\beta^{*}$ corresponds to the maximum inverse temperature [7]. The kinematical term $\tilde{B}$ and the dynamical term $\tilde{\tau}$ can be redefined so that their product remains unchanged. The different choices adopted lead to different values of the exponent $\alpha$ in eq. (6) and to different physical behaviour of the system through different partition functions. In this Letter we shall fix $\alpha=4$. This choice leads to normal behaviour of the system near the boundaries of the hadronic phase since it does not allow the energy density to become infinite, even for pointlike particles [6]. Consequently, for this choice, the maximum hadronic temperature is not a limiting temperature, since it can be reached at finite energy density and so it is consistent with a phase transition [8]. It also guarantees that the bootstrap singularity associated with a quark-hadron phase transition is reached in the thermodynamic limit [9].

\section{The existence of a critical point}

For the particular choice of $\alpha=4, \varphi$ assumes the form

$$
\varphi(\beta,\{\lambda\})=\frac{1}{2 \pi^{2} \beta B} \sum_{\mathrm{a}}\left(\lambda_{\mathrm{a}}(\{\lambda\})+\lambda_{\mathrm{a}}(\{\lambda\})^{-1}\right) \sum_{i} g_{\mathrm{a} i} m_{\mathrm{a} i}^{3} K_{1}\left(\beta m_{\mathrm{a} i}\right),
$$

where "a" represents a particular hadronic family and " $i$ " the hadrons in the family with different masses. The partition function of the pointlike interacting hadrons for this choice of $\alpha$ reads [7]:

$$
\begin{gathered}
\ln Z(V, \beta,\{\lambda\})=\sum_{b^{\prime}=-\infty}^{\infty} \lambda_{b}^{b^{\prime}} \sum_{q^{\prime}=-\infty}^{\infty} \lambda_{q}^{q^{\prime}} \sum_{s^{\prime}=-\infty}^{\infty} \lambda_{S}^{s^{\prime}} \sum_{\left|s^{\prime}\right|=0}^{\infty} \gamma_{s}^{\left|s^{\prime}\right|} \int \frac{2 V_{\mu} p^{\mu}}{(2 \pi)^{3}} \tilde{\tau}\left(p^{2}, b^{\prime}, q^{\prime}, s^{\prime},\left|s^{\prime}\right|\right) e^{-\beta^{\mu} p_{\mu}} d p^{4}= \\
\frac{4 B V}{\beta^{3}} \int_{\beta}^{\infty} x^{3} G(x,\{\lambda\}) d x \equiv \operatorname{Vf}(\beta,\{\lambda\})
\end{gathered}
$$

where the only left parameter $B$ is the energy density of the vacuum (bag constant). Including corrections due to the finite size of hadrons (Van der Waals volume corrections) one takes into account the repulsive part of the interaction [10]. With this improvement, the partition function in the grand canonical pressure ensemble, given by the Laplace transform of $Z(V, \beta,\{\lambda\})$, is written $[10]$

$$
\pi(\xi, \beta,\{\lambda\})=\left[\xi-f\left(\beta+\frac{\xi}{4 B},\{\lambda\}\right)\right]^{-1}
$$


where $f$ is the point-like partition function (8) divided by the volume and the Laplace variable $\xi$ gives a measure of the external forces acting on the system. In what follows we consider hadronic systems free of external forces and therefore we fix the variable $\xi$ at the value $\xi=0$ [9]. From eqs. (8) and (9) one obtains the energy and baryon number density $\left(\varepsilon, \nu_{b}\right)$ and the pressure $P$ of the thermodynamic system with the help of the following equations:

$$
\begin{gathered}
\varepsilon(\beta,\{\lambda\})=-\frac{\partial f}{\partial \beta}\left[1-\frac{1}{4 B} \frac{\partial f}{\partial \beta}\right]^{-1} \\
\nu_{b}^{(h)}(\beta,\{\lambda\})=\lambda_{b} \frac{\partial f}{\partial \lambda_{b}}\left[1-\frac{1}{4 B} \frac{\partial f}{\partial \beta}\right]^{-1} \\
P^{(h)}(\beta,\{\lambda\})=\frac{1}{\beta} f(\beta,\{\lambda\})\left[1-\frac{1}{4 B} \frac{\partial f}{\partial \beta}\right]^{-1}
\end{gathered}
$$

The onset of a phase transition can be traced by studying the pressure-volume isotherm curve (Fig. 1). It is obtained with the help of eqs. (10) and it reveals a region of instability where pressure and volume decrease together. This is a signal of a first-order phase transition and is due to the formation of bigger and bigger clusters as the hadronic matter approaches its boundaries. This instability is removed by a Maxwell construction as it is shown in Fig. 2 and discussed in more detail in what follows. As expected, no such behaviour is exhibited if the interaction incorporated in the statistical bootstrap hypothesis (SB) is neglected. In fact, in an ideal hadron gas (IHG), even with Van der Waals volume corrections, the pressure is everywhere a decreasing function of volume, keeping the system far from a phase transition. The results are displayed in Fig. 1 , where $\nu_{0}$ is the normal nuclear density $\nu_{0}=0.14 \mathrm{fm}^{-3}$. For the ideal hadron gas we have used in place of $f(\beta,\{\lambda\})$ the function:

$$
\frac{\ln Z_{I H G}(V, \beta,\{\lambda\})}{V}=\frac{1}{2 \pi^{2} \beta} \sum_{\mathrm{a}}\left[\lambda_{\mathrm{a}}(\{\lambda\})+\lambda_{\mathrm{a}}(\{\lambda\})^{-1}\right] \sum_{i} g_{\mathrm{a} i} m_{\mathrm{a} i} K_{2}\left(\beta m_{\mathrm{a} i}\right),
$$

where $g_{\mathrm{a} i}$ are degeneracy factors due to spin and isospin. In both cases (SB, IHG) we have imposed the constraints: $\langle S>=0$ (zero strangeness) and $\langle b>=2<q>$ (isospin symmetric system, i.e. the net number of $u$ and $d$ quarks are equal).

The existence of a maximal pressure in the isotherm curves (Fig. 1), obtained in the bootstrap solution (eqs. (10)), is the starting point in the search for the QCD critical point. In fact the end point of the critical line of a first order transition is reached when two isotherm 
curves corresponding to the two phases and the same temperature, intersect at the point of maximal pressure, on the hadronic side. The point of intersection, in this case, coincides with the critical point which in general corresponds to nonzero baryon chemical potential. In order to quantify the above description we have to consider the partition function of the quark-gluon system (QGP) and study the pressure-volume isotherm curves. For this purpose we adopt a minimal description of non interacting quarks and gluons within a bag supported by an external pressure $B$. More specifically, the free energy is written $[11]^{3}$

$$
\begin{aligned}
\beta^{-1} \ln Z_{Q G P}\left(V, \beta, \lambda_{u}, \lambda_{d}\right)= & \frac{N_{s} N_{c} V}{6 \pi^{2}} \sum_{i} \int_{0}^{\infty} \frac{p^{4}}{\sqrt{p^{2}+m_{i}^{2}}} \frac{1}{e^{\beta \sqrt{p^{2}+m_{i}^{2}}} \lambda_{i}^{-1}+1} d p \\
& +V \frac{8 \pi^{2}}{45} \beta^{-4}-B V .
\end{aligned}
$$

where the index $i$ runs over all quarks and antiquarks and $N_{s}, N_{c}$ refer to spin and colour degrees of freedom. The current masses are taken $m_{u} \approx 5.6 \mathrm{MeV}, m_{d} \approx 10 \mathrm{MeV}, m_{s} \approx$ $200 \mathrm{MeV}$ and the fugacities satisfy the constraints: $\lambda_{u}=\lambda_{\bar{u}}^{-1}, \lambda_{d}=\lambda_{\bar{d}}^{-1}, \lambda_{s}=\lambda_{\bar{s}}^{-1}=1$ (strangeness is set to zero). The first two terms in eq. (12) give the contribution to the free energy of the noninteracting quarks and gluons respectively and the third term gives the contribution of the vacuum (bag constant).

In order to study the $P-V$ isotherms of the quark-gluon system, we extract from the free energy (12) the baryon number density in quark matter, as follows

$$
\nu_{b}^{(q)}\left(\beta, \lambda_{u}, \lambda_{d}\right)=\frac{N_{s} N_{c}}{2 \pi^{2}} \sum_{i} \lambda_{i} N_{i} \int_{0}^{\infty} \frac{p^{2} d p}{e^{\beta \varepsilon_{i}}+\lambda_{i}} .
$$

The index $i$ includes only $u, \bar{u}$ and $d, \bar{d}$ quarks whereas $N_{i}= \pm 1$ for quarks $(u, d)$ and antiquarks $(\bar{u}, \bar{d})$ correspondingly. For a given value of $B$, the point where the hadron and quark phases meet can be traced on the $P-V$ isotherms for different temperatures. One has to require that at the same baryon density $\nu_{b}^{(q)}=\nu_{b}^{(h)}$ (eqs. (10) and (13)) the pressures, for the two phases, coincide. As can be seen in Fig. 2, for a low temperature, the quark matter isotherm meets the corresponding hadron isotherm at a point where the pressure is an increasing function of volume. This region is associated with the onset of a first-order

\footnotetext{
${ }^{3}$ The set of quark flavour fugacities $\left(\lambda_{u}, \lambda_{d}, \lambda_{s}, \gamma_{s}\right)$ can be used equivalently in the place of the set $\left(\lambda_{b}, \lambda_{q}, \lambda_{S}, \gamma_{s}\right)[7]$.
} 
phase transition and a Maxwell construction is needed in order to remove the instability $[6,9]$. As the temperature increases the quark matter isotherm meets the hadronic isotherm at a point which corresponds to a higher density and pressure. With this set of intersection points, the critical line of a first order quark-hadron phase transition is approximately built

up, in the phase diagram. At a certain temperature, the two phases meet at the point of maximal hadronic pressure (Fig. 2). The Maxwell construction is no longer needed since we have reached the end point of the critical line, in other words, we have reached a critical point of the strongly interacting matter. For higher temperatures we enter the crossover regime where quark and hadron phases are no longer distinguishable.

In Fig. 2 the limitations of our approach are also illustrated. In fact, in the absence of a final theory, based on QCD, which could provide us with a unified treatment of the equation of state in both phases, the matching of the isotherms, shown in Fig. 2, introduces artificial discontinuities in the derivatives for $T>T_{c}$, in a region where singularities are not allowed (crossover regime). Moreover, the scaling behaviour expected at the critical point and expressed through the isotherm critical exponent $(\delta)$, is not recovered at the point of intersection for $T=T_{c}$ (Fig. 2). Despite these drawbacks, our treatment concerning the existence and location of the critical point may still remain valid if in the exact theory of the equation of state, not only the unwanted discontinuities are washed out but, at the same time, the qualitative features of the hadronic isotherm, predicted by Statistical Bootstrap (Fig. 1), remain unchanged.

\section{The location of the critical point}

On the basis of the above mechanism and in order to locate the critical point in the phase diagram $\mu_{b}-T$, one has to determine the parameters $\left(\beta, \lambda_{u}, \lambda_{d}, \lambda_{s}, \lambda_{u}^{\prime}, \lambda_{d}^{\prime}\right)$ by solving the set of equations:

$$
\begin{gathered}
\nu_{b}^{(h)}\left(\beta, \lambda_{u}, \lambda_{d}, \lambda_{s}\right)=\nu_{b}^{(q)}\left(\beta, \lambda_{u}^{\prime}, \lambda_{d}^{\prime}\right) \\
P^{(h)}\left(\beta, \lambda_{u}, \lambda_{d}, \lambda_{s}\right)=P^{(q)}\left(\beta, \lambda_{u}^{\prime}, \lambda_{d}^{\prime}\right) \\
\frac{\partial P^{(h)}\left(\beta, \lambda_{u}, \lambda_{d}, \lambda_{s}\right)}{\partial \lambda_{u}}=0 \\
\left\langle S\left(\beta, \lambda_{u}, \lambda_{d}, \lambda_{s}\right)\right\rangle_{h}=0
\end{gathered}
$$




$$
\begin{gathered}
\left\langle b\left(\beta, \lambda_{u}, \lambda_{d}, \lambda_{s}\right)\right\rangle_{h}-2\left\langle Q\left(\beta, \lambda_{u}, \lambda_{d}, \lambda_{s}\right)\right\rangle_{h}=0 \\
\left\langle b\left(\beta, \lambda_{u}^{\prime}, \lambda_{d}^{\prime}\right)\right\rangle_{q}-2\left\langle Q\left(\beta, \lambda_{u}^{\prime}, \lambda_{d}^{\prime}\right)\right\rangle_{q}=0
\end{gathered}
$$

Equations (14d)-(14f) account for isospin symmetry and zero strangeness.

For a given value of the bag constant $B$ we have investigated the solution of eqs. (14) in order to build up the critical line (excluding eq. 14c) and locate the endpoint (including eq. 14c) in the phase diagram. We have projected out the solution onto the plane $\mu_{b}-T$ and our first remark is that for a wide range of values of the bag constant $B\left(B^{1 / 4}<282\right.$ $\mathrm{MeV}$ ) the critical baryon chemical potential $\mu_{c}$ is not zero (Fig. 3a). One may therefore establish, on the basis of the statistical bootstrap hypothesis, the existence of a critical point in hadronic matter at high temperatures and nonzero baryon chemical potential. A typical solution is obtained if we impose the condition $\mu_{b}$ (hadronic matter $)=\mu_{b}$ (quark matter) at the critical point. The solution obtained in this case corresponds to the value $B^{1 / 4}=250$ $\mathrm{MeV}$ and it is illustrated (SB) in Fig. 3b. The location of the endpoint (critical point) in this solution is fixed by the critical values $T_{c} \approx 171 \mathrm{MeV}$ and $\mu_{c} \approx 385 \mathrm{MeV}$ whereas the critical temperature at zero chemical potential (at the end of the crossover) is $T_{0} \approx 188 \mathrm{MeV}$. In the same figure, the solution of lattice QCD with $n_{f}=2+1$ (LQCD), corresponding to the critical values $T_{c}=160 \pm 3.5 \mathrm{MeV}, \mu_{c}=725 \pm 35 \mathrm{MeV}$ and $T_{0}=172 \mathrm{MeV}$ is also shown [2] for comparison. The discrepancy of these two solutions is due (a) to the fact that the lattice QCD result for the critical point is based on rather unphysical input values of the quark masses $m_{u}, m_{d}$ and (b) to the uncertainties in the partition function (12) coming from neglected interactions in the quark-gluon system. One expects that, when both, the quark masses get reduced and the quark-gluon pressure for nonzero chemical potential becomes available on the lattice [12], the SB and LQCD critical lines in Fig. 3b will converge to a unique solution, supported both by QCD and Statistical Bootstrap. Finally, it is of interest to note that within a different frame of approximate theories (Nambu-Jona-Lasinio (NJL) model or a random matrix (RM) approach) the QCD critical point has been located in the region $T_{c} \approx 100 \mathrm{MeV}, \mu_{c} \approx 600-700 \mathrm{MeV}$ of the phase diagram [1], in a good distance from the SB and LQCD solutions (Fig. 3b).

In Fig. $3 b$ the freeze-out points associated with recent heavy-ion experiments are also shown on the plane $\mu_{b}-T$ [13]. One observes that the SB solution brings the critical 
point close to the freeze-out area of the SPS experiments [13]. However, before drawing any conclusion concerning the phenomenology of the SB critical point, an improved lattice QCD calculation of the pressure of the quark-gluon system with small quark masses is needed [12].

In conclusion, we have shown that the hadronic matter develops a critical point at high temperatures and nonzero baryon chemical potential, in accordance with the requirements of the QCD phase diagram. Our approach was based on the Hagedorn Statistical Bootstrap Principle (SB) as it was elaborated, in connection with quark-hadron phase transition, in references [6,9]. With the present degree of approximation for the quark-gluon partition function, the location of the SB critical point, in the phase diagram, was found close to the freeze-out points of the SPS experiments with nuclei $\left(T_{c} \approx 171 \mathrm{MeV}, \mu_{c} \approx 385 \mathrm{MeV}\right)$.

\section{Acknowledgements}

It is a pleasure to thank Fotis Diakonos for fruitful discussions. This work was supported in part by the Research Committee of the University of Athens.

\section{References}

[1] F. Wilczek, hep-ph/0003183;

J. Berges, K. Rajagopal, Nucl. Phys. B 538 (1999) 215;

M. A. Halasz, A. D. Jackson, R. E. Shrock, M. A. Stephanov, J. J. Verbaarschot, Phys. Rev. D 58 (1998) 096007

[2] Z. Fodor, S. D. Katz, hep-lat/0106002

[3] N. G. Antoniou, "Multiparticle production in chiral QCD phase transition", Proceedings of the XV International Symposium on Multiparticle Dynamics, Stara Lesna, Slovakia 12-16 September 1995, editors: D. Bruncko, L. Sandor, J. Urban, World Scientific (1996) p. 371; "Fluctuations in Chiral Condensation", Proceedings of the VII Workshop on Multiparticle Production (Correlations and Fluctuations) Nijmegen, June 30 - July 6, 1996, editors: R. C. Hwa, W. Kittel, W. J. Metzger, D. J. Schotanus, World Scientific (1997) p. 160;

N. G. Antoniou, F. K. Diakonos, C. N. Ktorides, M. Lahanas, Phys. Lett. B 432 (1998) 
8

M. A. Stephanov, K. Rajagopal, E. V. Shuryak, Phys. Rev. Lett. 81 (1998) 4816;

M. A. Stephanov, K. Rajagopal, E. V. Shuryak, Phys. Rev. D 60 (1999) 114028;

N. G. Antoniou, Y. F. Contoyiannis, F. K. Diakonos, Nucl. Phys. A 661 (1999) 399c;

E. V. Shuryak, M. A. Stephanov, Phys. Rev. C 63 (2001) 064903;

N. G. Antoniou, Y. F. Contoyiannis, F. K. Diakonos, A. I. Karanikas, C. N. Ktorides, Nucl. Phys. A 693 (2001) 799

[4] R. Hagedorn, Nuovo Cimento Suppl. III, (1965) 147;

R. Hagedorn, I. Montvay, J. Rafelski, "Hadronic Matter at Extreme Energy Density", eds. N. Cabbibo and L. Sertorio, Plenum Press, New York, (1980) 49

[5] R. Hagedorn, J. Rafelski, Phys. Lett. B 97 (1980) 136

[6] R. Fiore, R. Hagedorn, F. d' Isep, Nuovo Cimento A 88 (1985) 301

[7] A. S. Kapoyannis, C. N. Ktorides, A. D. Panagiotou, J. Phys. G 23 (1997) 1921; Phys. Rev. D 58 (1998) 034009; Phys. Rev. C 58 (1998) 2879; Eur. Phys. J. C 14 (2000) 299

[8] N. Cabbibo, G. Parisi, Phys. Lett. B 59 (1975) 67

[9] J. Letessier, A. Tounsi Nuovo Cimento A 99 (1988) 521

[10] R. Hagedorn, Z. Phys. C 17 (1983) 265

[11] J. Rafelski, R. Hagedorn: "From hadron gas to quark matter II" in Statistical mechanics of quarks and hadrons edited by H. Satz (North Holland Publ. Co., Amsterdam, 1981)

[12] F. Karsch, E. Laermann, A. Peikert, Phys. Lett. B 478 (2000) 447;

Z. Fodor, S. D. Katz, K. K. Szabó, hep-lat/0208078

[13] P. Braun-Munzinger, J. Stachel, J. Phys. G 28 (2002) 1971

\section{Figure Captions}

Fig. 1 The pressure-volume isotherms for hadronic matter described by Statistical Bootstrap (SB) and for the ideal hadron gas (IHG). 
Fig. 2 The intersection points of the pressure-volume isotherms of the two phases are shown, for different temperatures, and the Maxwell construction associated with the phase transition is illustrated.

Fig. 3 (a) The SB solutions for the critical line and the corresponding critical point, for different choices of the bag constant. (b) A typical SB solution is shown in the phase diagram $\left(\mu_{b}-T\right)$ together with the LQCD solution and the freeze-out points of heavy-ion experiments. Also the NJL-RM solution for the location of the critical point is shown. 


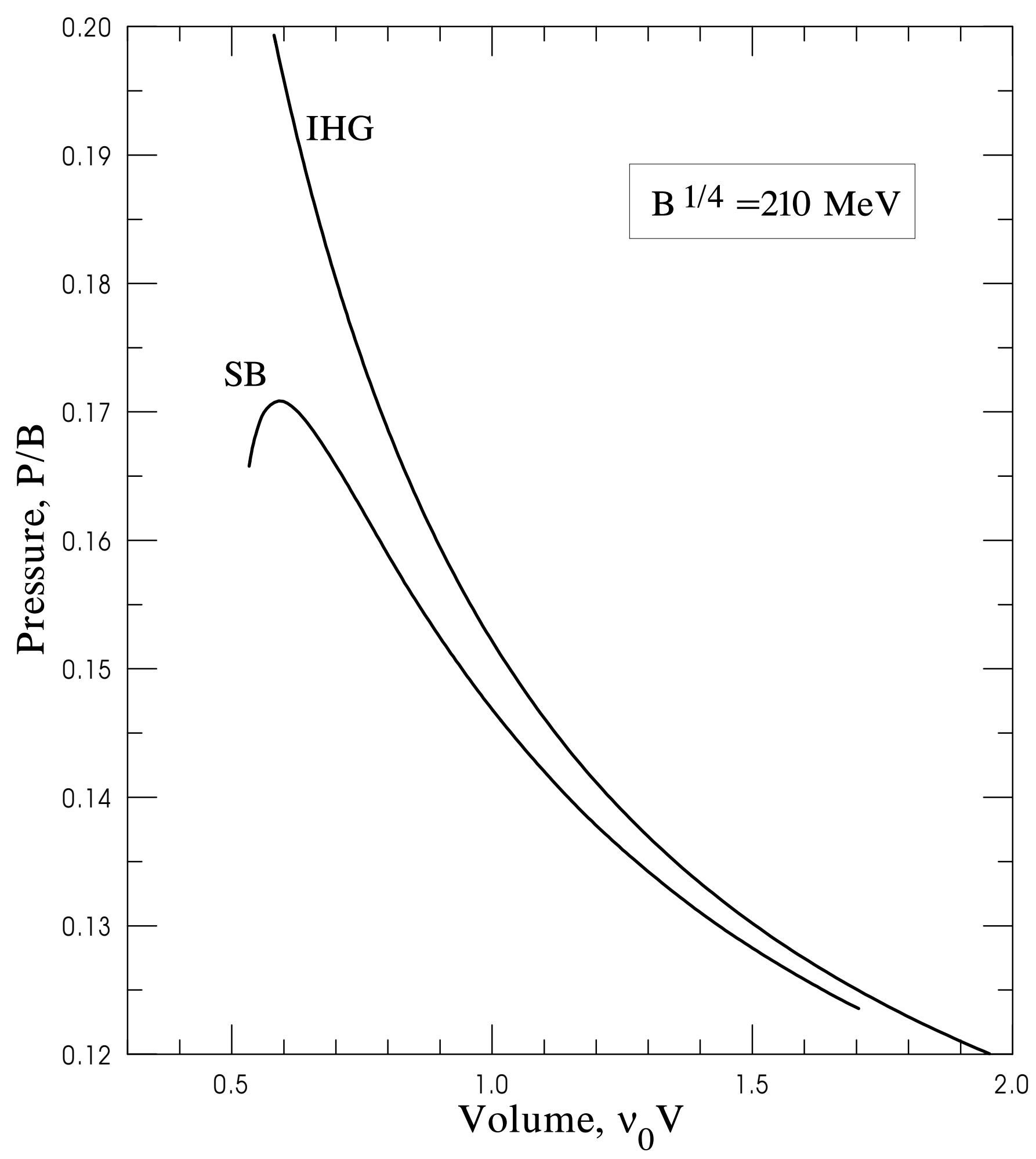




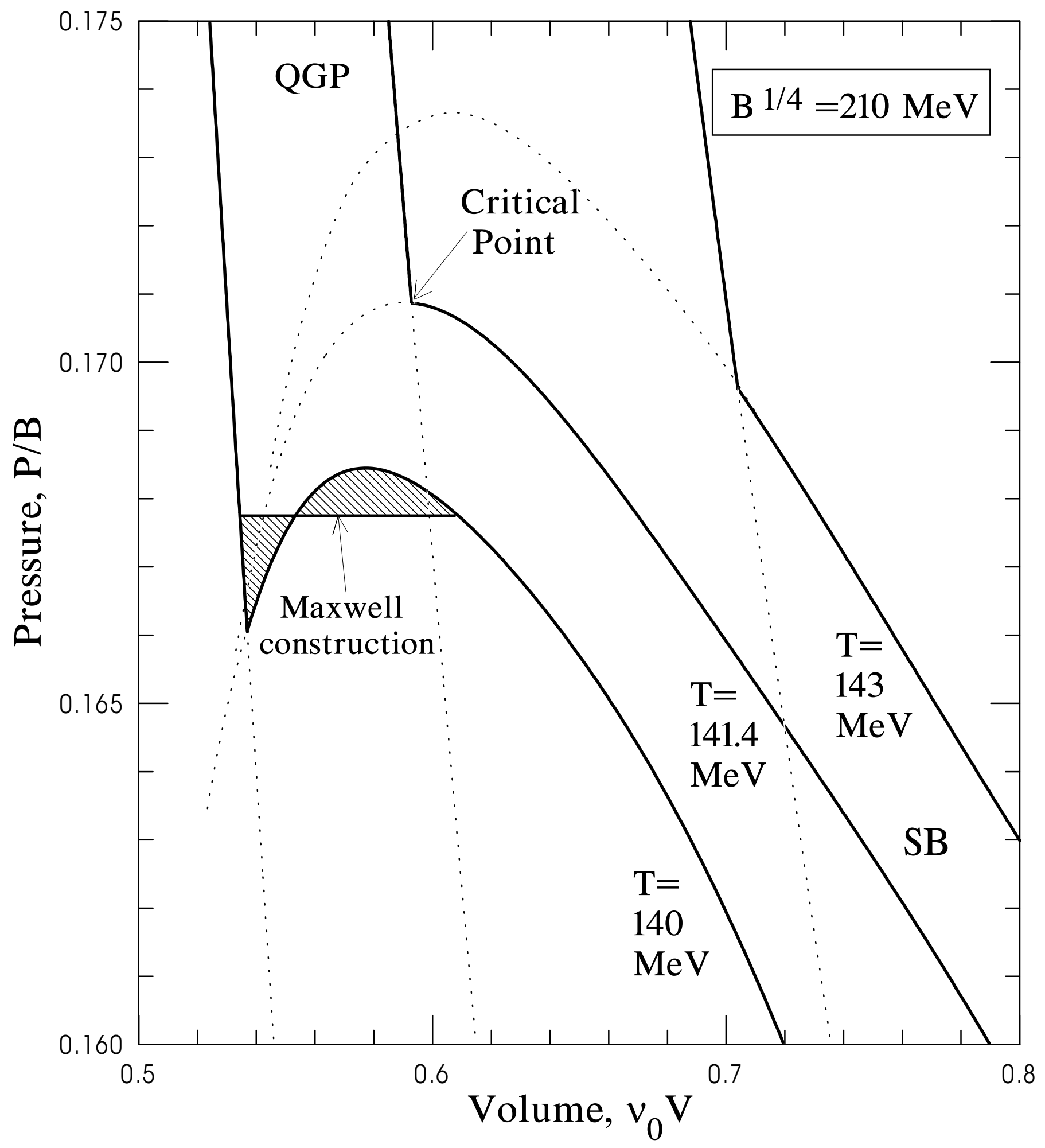



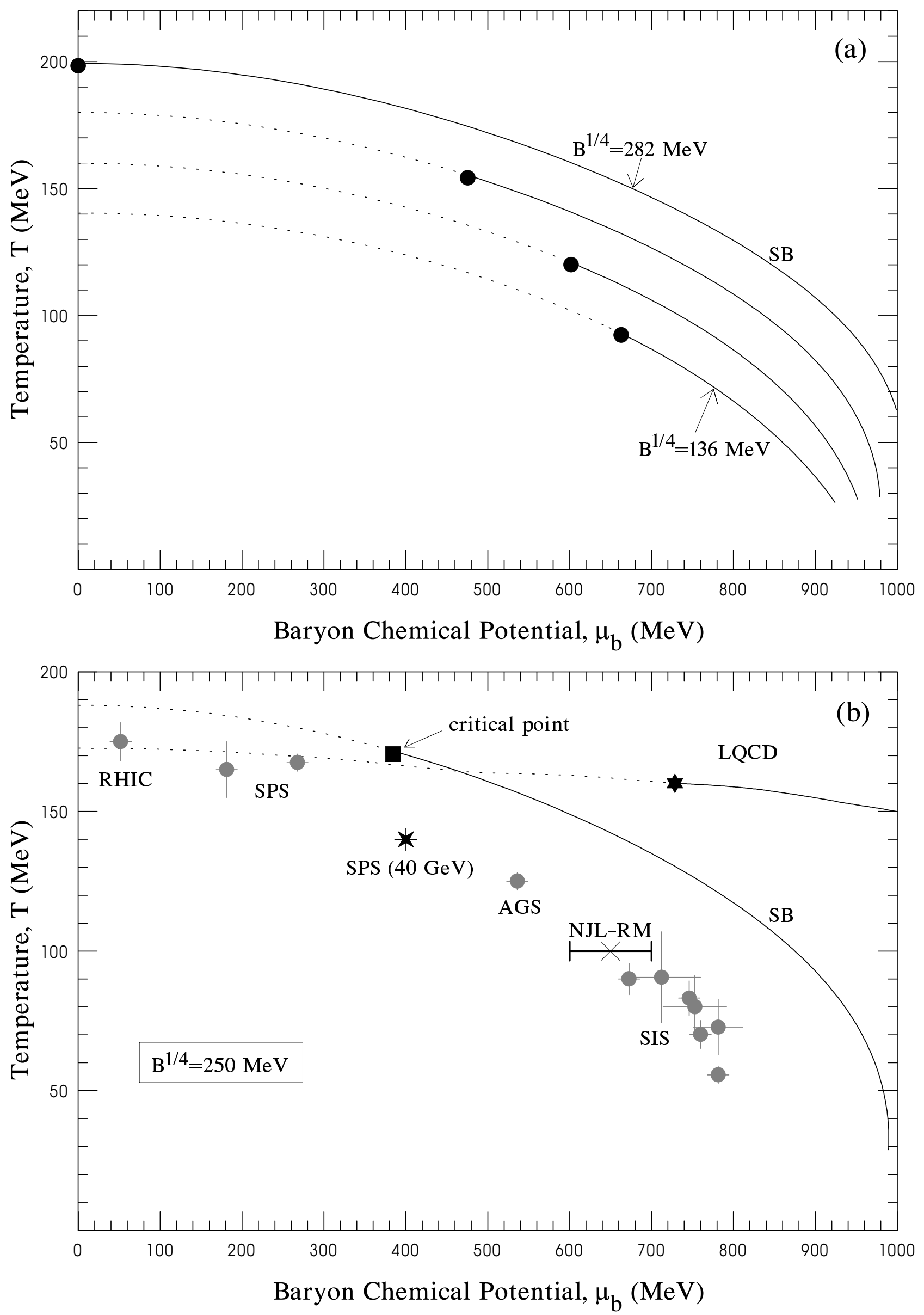